\title{
P(III) vs. P(V): A P(V) Reagent for Thiophosphoramidate Linkages and Application to An Asymmetric Synthesis of a Cyclic Dinucleotide STING Agonist
}

Bin Zheng*, Chao Hang, Jason Zhu, Geoffrey E. Purdum, Melda Sezen-Edmonds, Daniel S. Treitler, Miao Yu, Changxia Yuan, Ye Zhu, Adam Freitag, Siwei Guo, Guanghui Zhu, Ben Hritzko, James Paulson, Jonathan G. Shackman, Brian L. He, Weiqing Fu, Hua Chia Tai, Sloan Ayers, Hyunsoo Park, Martin D. Eastgate, Ben Cohen, Amanda Rogers, Qinggang Wang, Michael A. Schmidt*

\section{Bin.Zheng@bms.com, Michael.Schmidt@bms.com}

Bristol Myers Squibb, Chemical Process Development, 1 Squibb Dr. New Brunswick, New Jersey 08903.

Abstract: A highly stereoselective synthesis of a cyclic dinucleotide (CDN) STING agonist containing two chiral thiophosphoramidate linkages is described. These rare, yet key functional groups were, for the first time, installed efficiently and with high diastereoselectivity using a specially designed $\mathrm{P}(\mathrm{V})$ reagent. By utilizing this strategy, the $\mathrm{CDN}$ was prepared in greater than sixteen-fold higher yield than the prior P(III) approach, with fewer hazardous reagents and chromatographic purifications.

Agonists of the stimulator of interferon genes (STING) are of interest as potential oncology therapeutics. ${ }^{1} \quad$ A common structural feature in these compounds is a cyclic dinucleotide $(\mathrm{CDN})$ macrocycle, similar to the natural ligand cyclic GMP-AMP (cGAMP, Figure 1A). While the linchpin phosphorus atoms in cGAMP are not stereogenic centers, in vivo stability-enhancing modifications ${ }^{2}$ such as monosulfurization result in stereogenic phosphorus centers with limited means of synthesis. Our target, CDN 1 (Figure 1A), not only contained these stereogenic, thiolated phosphorus centers, but also contained phosphorus-nitrogen linkages, which dramatically altered the chemistry involved in the synthesis. The initial synthesis of $\mathbf{1}$ using stereouncontrolled $\mathrm{P}(\mathrm{III})$ phosphoramidite chemistry was plagued with difficulties (Figure 1B) and would not be suitable for further evaluation, necessitating a new synthetic route. While there have been recent advances in the asymmetric synthesis of thiophosphate diester CDNs, such as those from ourselves ${ }^{3}$ and Leahy \& Miller ${ }^{4}$, there is no approach for the asymmetric synthesis of the more challenging thiophosphoramidic acid derived compounds. Herein we describe an asymmetric, $\mathrm{P}(\mathrm{V})$ reagent-based approach to thiophosphoramidate CDN 1 that avoided the limitations encountered in the $\mathrm{P}(\mathrm{III})$ synthesis (Figure 1C). 

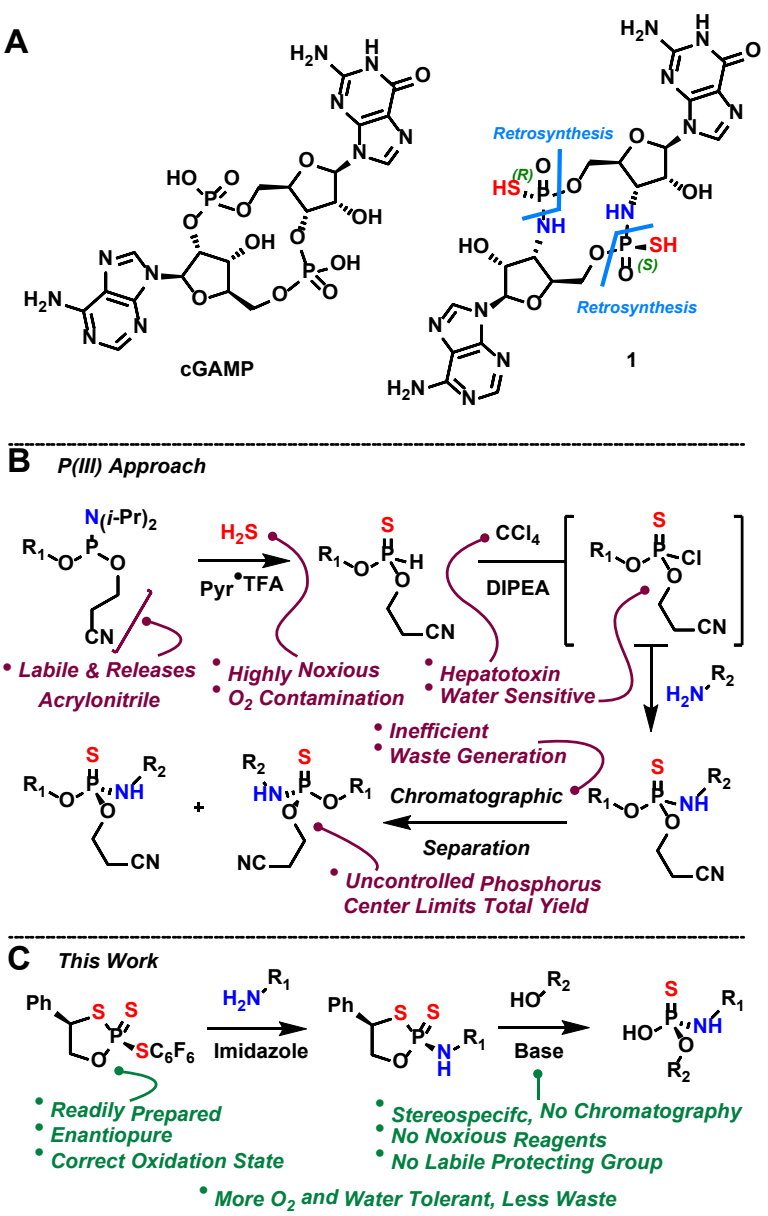

Figure 1. A) cGAMP and target 1. B) P(III) method to prepare thiophosphoramidate linkages C) This work, development of a $\mathrm{P}(\mathrm{V})$-reagent approach.
The previous route began with the 3-azanucleoside $\mathbf{2}$ and employed standard P(III) phosphoramidite chemistry (Scheme 1$)^{5}$ which was successful for producing small quantities of 1. However, we discovered crippling challenges in using this route to deliver material to enable the study of the clinical efficacy of $\mathbf{1}$, all of which stemmed from the introduction of the chiral thiophosphoramidate. For example (Figure 1B), the P(III) phosphoramidite chemistry was highly sensitive to hydrolysis. The sulfur was added using gaseous hydrogen sulfide which, besides its significant odor and toxicity, must be strictly controlled for impurities (adventitious oxygen forms phosphoramidate analogues). The final oxidation used an excess of highly toxic carbon tetrachloride to form a moisture-sensitive thiophosphoryl chloride. Lastly, and most significantly, the coupling sequence was not stereocontrolled, dramatically reducing the yield and necessitating large-scale reversed-phase chromatography to separate the phosphorus diastereomers. As a result, the coupling of the two nucleosides with concomitant formation of the phosphorus stereocenters had multiple, complex issues and led to an extremely low overall yield of $1(0.6 \%) .{ }^{5}$

\section{Scheme 1}

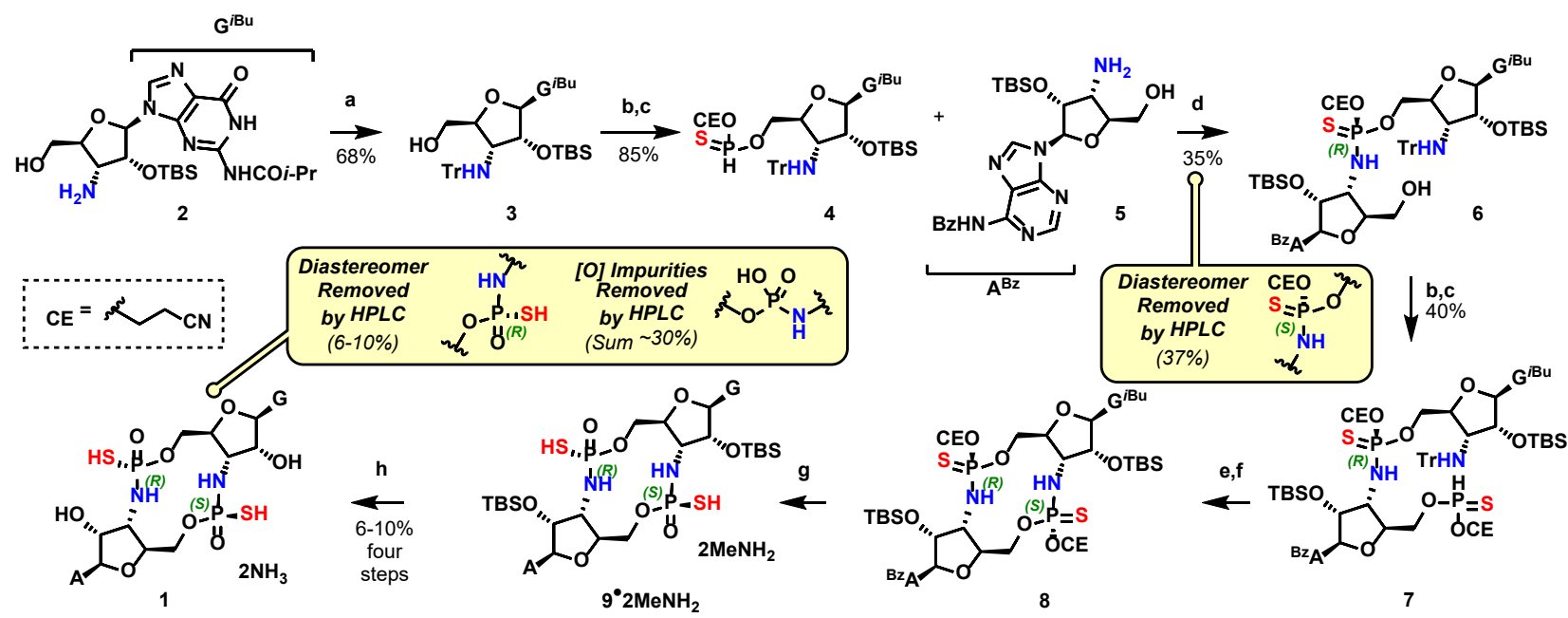

a) $\mathrm{TrCl}, \mathrm{Et} 3 \mathrm{~N}$, pyridine, b) 2-cyanoethyl $N, N, N^{\prime}, N^{\prime}$-tetraisopropylphosphorodiamidite, Pyr•TFA, c) $\mathrm{H}_{2} \mathrm{~S}, \mathrm{Pyr}^{\bullet} \mathrm{TFA}$ d) $\mathrm{CCl} 4$ then 5 , DIPEA, e) dichloroacetic acid, DCM/water, f) $\mathrm{CCl}_{4}$ then $\left.\left.\mathrm{Et}_{3} \mathrm{~N}, \mathrm{~g}\right) \mathrm{MeNH}_{2}, \mathrm{MeOH}, \mathrm{h}\right) \mathrm{Et}_{3} \mathrm{~N} \cdot 3 \mathrm{HF}, \mathrm{Et}_{3} \mathrm{~N}$, pyridine. 
In light of these challenges, we embarked on a new synthetic strategy that could simultaneously address all of these concerns. In particular, we desired a diastereoselective synthesis that: 1) delivered the correct stereochemical configuration at phosphorus, to improve yield and minimize chromatographic purifications, 2) was performed at the correct oxidation state, to avoid the use of noxious hydrogen sulfide gas and toxic carbon tetrachloride, and 3) managed the unique reactivity and stability challenges of the thiophosphoramidate linkages. To achieve the aforementioned goals, we explored a $\mathrm{P}(\mathrm{V})$ reagent-based approach (the "PSI-platform") that we invented, in collaboration with the Baran laboratory, and recently disclosed in the diastereoselective synthesis of phosphorothioate diesters. ${ }^{3}$

Preliminary work using PSI indicated that the thiophosphoramidate linkage would produce some unique challenges in terms of reactivity and stability. For example, the sequence of addition was vital. The reaction of a PSI reagent with the amine first, followed by an alcohol was much higher yielding than the alternative (i.e. reacting a PSI reagent with an alcohol, then an amine). We found that we could form the first thiophosphoramidate linkage (Figure 2, $\mathbf{1 0}$ or 11) with PSI reagents derived from either cis-limonene oxide Error! Bookmark not defined. (12) or trans-limonene oxide ${ }^{6}(\mathbf{1 3})$, our previously disclosed reagents, though they did not react further with the amine of $\mathbf{1 0}$ or $\mathbf{1 1}$ (Figure 2, Top). We reasoned that this was due to the steric requirements of these reactants. Thus we explored the development of a new sub-class of reagents with a smaller steric footprint and higher reactivity. After screening a variety of enantiopure epoxides (14-17 Figure 2, Bottom), we focused on a reagent derived from styrene oxide (17), of which both enantiomers are inexpensive and readily available. The resulting optically pure trithiophosphate reagents, $\mathbf{1 8}$ and its enantiomer $\mathbf{2 1}$ (vide infra), are stable, crystalline and straightforward to prepare (Figure
2

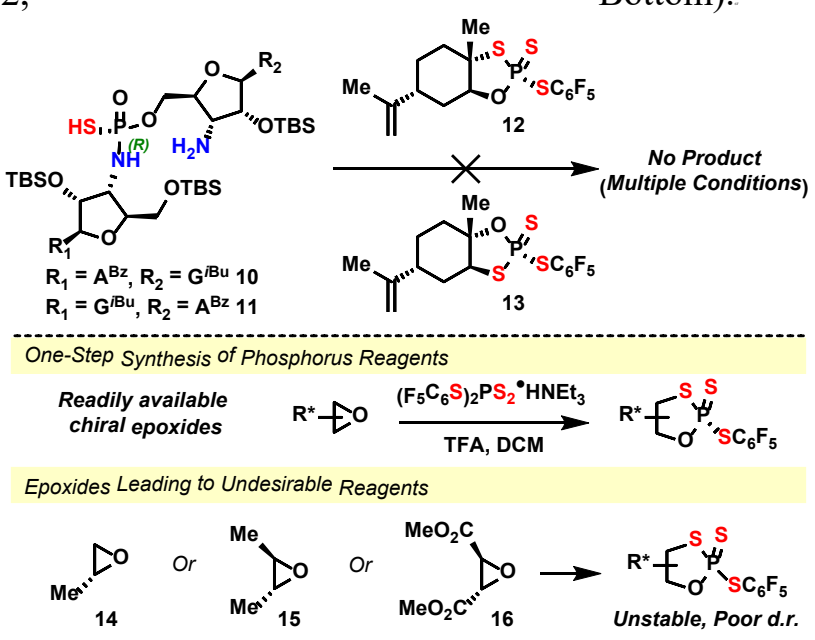

Styrene Oxide Reagent

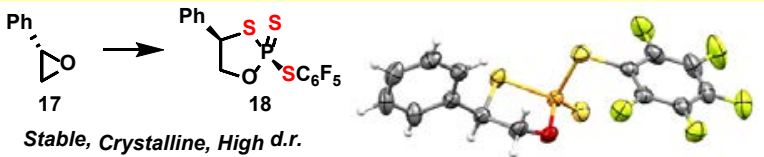

Figure 2. Unsuccessful reaction of PSI reagents with hindered amine $\mathbf{1 0}$ or $\mathbf{1 1}$ (top). Examination of alternative PSI reagents and discovery of a styrene-oxide derived reagent $\mathbf{1 8}$ (bottom).

We discovered that these PSI reagents reacted with 5 preferentially at the 5' hydroxyl group rather than the desired 3' amine, thus we needed to protect the 5' hydroxyl group (Scheme 2). This was readily achieved by the treatment of 5 with $\mathrm{TBSCl}$ in the presence of imidazole, which afforded the TBS ether product directly as the amine hydrochloride salt, and prevented the migration of the purine benzoyl group to the primary amine (a side reaction observed with stronger bases). After free-basing, the bis-TBS compound 19 was isolated by crystallization from heptane and MTBE in 76\% yield. The reaction between 18 and 19 was now straightforward, affording 20 in 83\% yield (typically 50:1 d.r. at phosphorus). By taking advantage of the affinity of the phosphorus center of $\mathbf{2 0}$ towards oxygen nucleophiles ${ }^{8}$, we attempted to take $\mathbf{2}$ directly into the reaction without protecting its primary amine. This coupling proceeded most effectively by the use of sodium tert-amylate in THF, affording the linear dinucleoside with $69 \%$ isolated yield and excellent control of the 
phosphorus stereocenter (crude d.r. is typically 250:1). Of note, while the protecting groups on the purine bases (i.e., benzoyl group on the adenine and the iso-butyryl group on the guanine) were not needed to control chemoselectivity in the synthesis, we found the unprotected molecules were poorly soluble in most organic solvents and, as a result, reactions were difficult to control. We therefore opted to maintain bases protected.

To create the next phosphorus stereocenter, we needed to use enantiomeric PSI reagent 21. Gratifyingly, the reaction between $\mathbf{1 0}$ and the styrene oxide-derived reagent $\mathbf{2 1}$ was successfully conducted in a similar fashion to $\mathbf{1 9}$ with imidazole as the base to afford 22 (typically 50:1 d.r. at the new phosphorus center). After an aqueous work up, addition of dichloroacetic acid selectively deprotected the primary TBS group, freeing up the primary alcohol which would be used to close the macrocycle. After a second aqueous workup, the product $\mathbf{2 3}$ was precipitated out in approximately $50 \%$ purity and $60 \%$ corrected yield. Further purification was not required.

\section{Scheme 2}

The macrocyclization was explored under a variety of conditions, and $\mathrm{LiO} t-\mathrm{Bu}$ in a mixture of anhydrous NMP and THF proved optimal. As in the previous P-O bond-forming step (i.e., to form 10), the crude d.r. of the newly formed phosphorus stereocenter was extremely high (typically 150:1). Initially, the macrocyclization was performed at a concentration of $4.0 \mathrm{mM}$ in NMP/THF; however, we found we could significantly increase the concentration to $64 \mathrm{mM}$ without detriment. After quenching with acetic acid, diluting with MTBE, and an aqueous lithium chloride workup, macrocycle $\mathbf{2 4}$ was obtained in $70 \%$ yield, able to be used directly in the subsequent reaction.

Next, the purine protecting groups of $\mathbf{2 4}$ were removed with methylamine and the product isolated by crystallization in $76 \%$ yield with $99.5 \%$ purity. Finally, the TBS groups were removed with $\mathrm{Et}_{3} \mathrm{~N} \cdot 3 \mathrm{HF}$, buffered by additional triethylamine in THF. After the reaction was complete, crude $\mathbf{1}$ was precipitated with acetonitrile and then purified by the only reversed-phase chromatography in the sequence to afford the product in $70 \%$ yield.

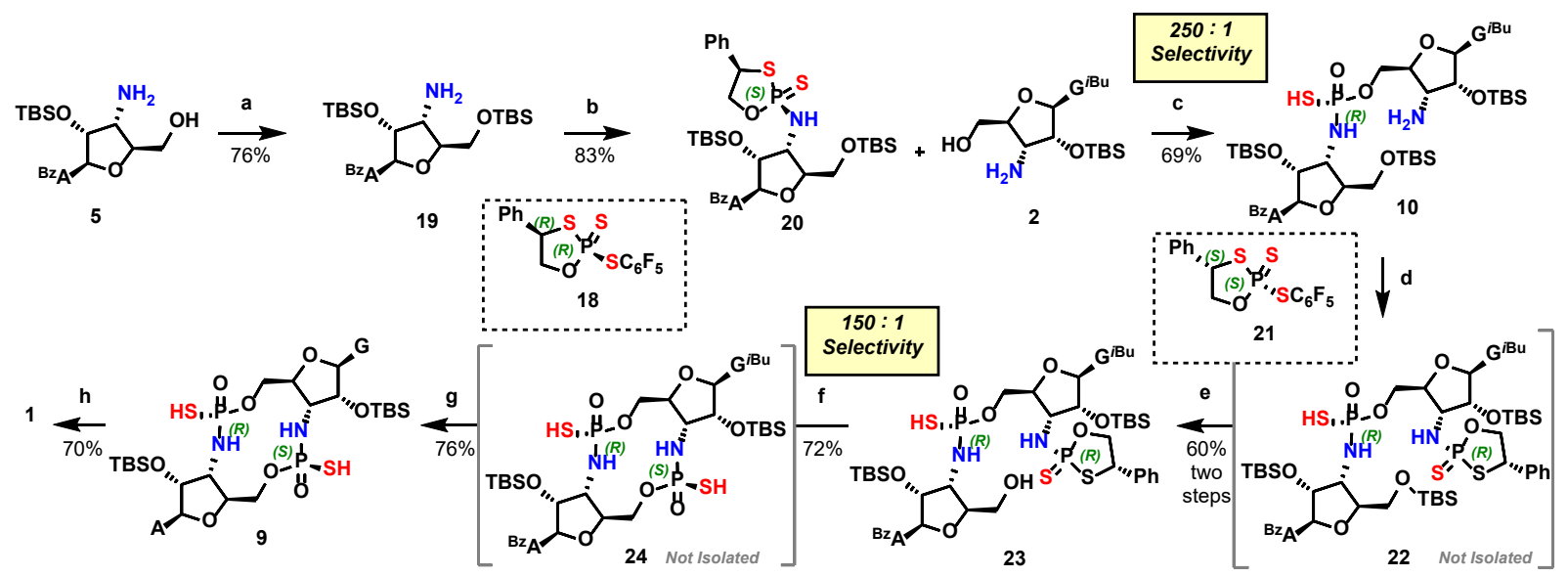

a) $\mathrm{TBSCl}$, imidazole, b) 18, imidazole, c) $\mathrm{NaO} t$-Am, THF, d) 21, imidazole, 2-MeTHF, e) dichloroacetic acid, f) $\mathrm{LiO} t$-Bu, $\mathrm{THF} / \mathrm{NMP}, \mathrm{g}) \mathrm{MeNH}_{2}$, h) $\mathrm{Et}_{3} \mathrm{~N} \cdot 3 \mathrm{HF}, \mathrm{Et}_{3} \mathrm{~N}, \mathrm{THF}$.

In conclusion, we have invented a new reagent for the asymmetric synthesis of phosphoramidic acid derivatives. This reagent enabled the synthesis of CDN 1 in $10 \%$ total yield, a more than a 16-fold improvement versus the prior P(III) approach. By utilizing a reagent at the correct oxidation state, in place of a traditional P(III) approach, the aza-nucleosides 
could be coupled at $\mathrm{P}(\mathrm{V})$ for the first time. This eliminated the need for toxic, odoriferous hydrogen sulfide and the formation of difficult to purge phosphorus-oxygen impurities. Critically, the $\mathrm{P}(\mathrm{V})$ approach proved highly stereoselective, which contributed to the overall higher yield, and eliminated an early-stage preparative reversedphase chromatographic purification. This route successfully enabled production of material to support the clinical efficacy study. Further efforts focused on identifying and understanding the critical factors that enabled the successful execution of this sequence at multi-kilogram scale will be reported in due course.

\section{Author Information}

Corresponding authors

Bin Zheng-Bristol Myers Squibb, Chemical Process Development, New Brunswick NJ, 08903

Michael A. Schmidt-Bristol Myers Squibb, Chemical Process Development, New Brunswick NJ, 08903

\section{Authors}

Chao Hang-Bristol Myers Squibb, Chemical Process Development, New Brunswick NJ, 08903

Jason Zhu-Bristol Myers Squibb, Chemical Process Development, New Brunswick NJ, 08903

Geoffrey E. Purdum-Bristol Myers Squibb, Chemical Process Development, New Brunswick NJ, 08903

Melda Sezen-Edmonds-Bristol Myers Squibb, Chemical Process Development, New Brunswick NJ, 08903

Daniel S. Treitler-Bristol Myers Squibb, Chemical Process Development, New Brunswick NJ, 08903

Miao Yu-Bristol Myers Squibb, Chemical Process Development, New Brunswick NJ, 08903

Changxia Yuan-Bristol Myers Squibb, Chemical Process Development, New Brunswick NJ, 08903
Ye Zhu-Bristol Myers Squibb, Chemical Process Development, New Brunswick NJ, 08903

Adam Freitag-Bristol Myers Squibb, Chemical Process Development, New Brunswick NJ, 08903

Siwei Guo-Bristol Myers Squibb, Chemical Process Development, New Brunswick NJ, 08903

Guanghui Zhu-Bristol Myers Squibb, Chemical Process Development, New Brunswick NJ, 08903

Ben Hritzko-Bristol Myers Squibb, Chemical Process Development, New Brunswick NJ, 08903

James Paulson-Bristol Myers Squibb, Chemical Process Development, New Brunswick NJ, 08903

Jonathan G. Shackman-Bristol Myers Squibb, Chemical Process Development, New Brunswick NJ, 08903

Brian L. He-Bristol Myers Squibb, Chemical Process Development, New Brunswick NJ, 08903

Weiqing Fu-Bristol Myers Squibb, Chemical Process Development, New Brunswick NJ, 08903

Hua Chia Tai-Bristol Myers Squibb, Chemical Process Development, New Brunswick NJ, 08903

Sloan Ayers-Bristol Myers Squibb, Chemical Process Development, New Brunswick NJ, 08903

Hyunsoo Park-Bristol Myers Squibb, Chemical Process Development, New Brunswick NJ, 08903

Martin D. Eastgate-Bristol Myers Squibb, Chemical Process Development, New Brunswick NJ, 08903

Ben Cohen-Bristol Myers Squibb, Chemical Process Development, New Brunswick NJ, 08903

Amanda Rogers-Bristol Myers Squibb, Chemical Process Development, New Brunswick NJ, 08903

Qinggang Wang-Bristol Myers Squibb, Chemical Process Development, New Brunswick NJ, 08903 


\section{Author Contributions}

The manuscript was written through contributions of all authors. All authors have given approval to the final version of the manuscript.

\section{Notes}

A patent application is pending (WO 202000/117739 A1).

\section{Acknowledgements}

We would like to thank Elizabeth Yuill, Yehia Baghdady and Ziqing Lin for assistance with analytical measurements, Amy A. Sarjeant for assistance preparing crystallographic files and Professor Phil Baran for assistance reviewing the manuscript.

Accession Code CCDC 2065361 contains the supplementary crystallographic data for this paper. These data can be obtained free of charge via www.ccdc.cam.ac.uk/data request/cif, or by emailing data_re-quest@ccdc.cam.ac.uk, or by contacting The Cambridge Crystallographic Data Centre, 12 Union Road, Cambridge CB2 1EZ, UK; fax: +44 1223336033 . 
${ }^{1}$ a) Corrales, L.; Gajewski, T. F. Molecular Pathways: Targeting the Stimulator of Interferon Genes (STING) in the Immunotheraphy of Cancer. Clin. Cancer Res. 2015, 21, 4774. b) Marloye, M.; Lawler, S. E.; Berger, G. Current Patent and Clinical Status of Stimulator of Interferon Genes (STING) Agonists for Cancer Immunotherapy. Pharm. Pat. Anal. 2019, 8, 87.

2 a) Vosberg, H. P.; Eckstein F. Effect of Deoxynucleoside Phosphorothioates Incorporated in DNA on Cleavage by Restriction Enzymes. J. Bio. Chem. 1982, 257, 6595. b) Purcell, J.; Hengge, A. C. The Thermodynamics of Phosphate Versus Phosphorothioate Ester Hydrolysis. J. Org. Chem. 2005, 70, 8437.

${ }^{3}$ Knouse, K. W.; deGruyter, J. N.; Schmidt, M. A.; Zheng, B.; Vantourout, J. C.; Kingston, C.; Mercer, S. E.; Mcdonald, I. M.; Olson, R. E.; Zhu, Y.; Hang, C.; Zhu, J.; Yuan, C.; Wang, Q.; Park, P.; Eastgate, M. D.; Baran, P. S. Unlocking P(V): Reagents for Chiral Phosphorothioate Synthesis. Science, 2018, 361, 1234.

${ }^{4}$ Featherston, A. L.; Kwon, Y.; Pompeo, M. M.; Engl, O. D.; Leahy, D. K.; Miller, S. J. Catalytic Asymmetric and Stereodivergent Oligonucleotide Synthesis. Science, 2021, 371, 702.

${ }^{5}$ Glick, G.; Ghosh, S.; Olhava, E. J.; Roush, W. R.; Jones, R. Cyclic Dinucleotides for Treating Conditions Associated with STING Activity Such as Cancer. 2017, WO 2017/123669 A1.

${ }^{6}$ Xu, D.; Rivas-Bascón, N.; Padial, N. M.; Knouse, K. W.; Zheng, B.; Vantourout, J. C.; Schmidt, M. A.; Eastgate, M. D.; Baran, P. S. Enantiodivergent Formation of C-P Bonds: Synthesis of P-Chiral Phosphines and Methylphosphonate Oligonucleotides. J. Am. Chem. Soc. 2020, 142, 5785.

${ }^{7}$ Please see the Supporting Information for details.

${ }^{8}$ Vantourout, J. C.; Adusumalli, S. R.; Knouse, K. W.; Flood, D. T. ; Ramirez, A.; Padial, N. M.; Istrate, A.; Maziarz, K.; deGruyter, J. N.; Merchant, R. R.; Qiao, J. X.; Schmidt, M. A.; Deery, M. J.; Eastgate, M. D.; Dawson, P. E.; Bernardea, G. J. L.; Baran, P. S. Serine-Selective Bioconjugation. J. Am. Chem. Soc. 2020, 142, 17236. 